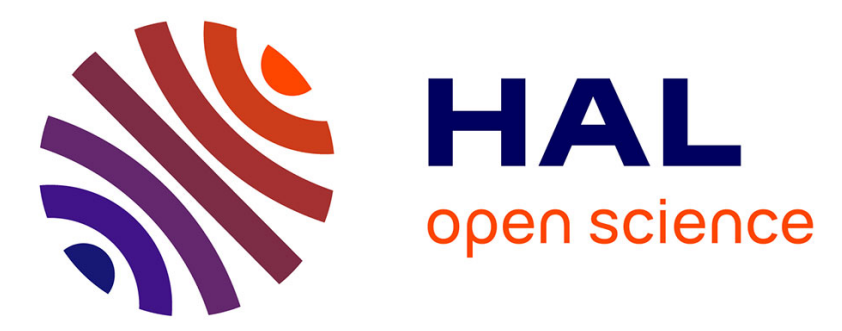

\title{
Elastic surface waves in crystals. Part 1: Review of the physics
}

Nathalie Favretto-Cristini, Dimitri Komatitsch, Carcione M. José, Cavallini

Fabio

\section{- To cite this version:}

Nathalie Favretto-Cristini, Dimitri Komatitsch, Carcione M. José, Cavallini Fabio. Elastic surface waves in crystals. Part 1: Review of the physics. Ultrasonics, 2011, 51 (6), pp.653-660. 10.1016/j.ultras.2011.02.007 . hal-00598334

\section{HAL Id: hal-00598334 \\ https://hal.science/hal-00598334}

Submitted on 29 Jan 2014

HAL is a multi-disciplinary open access archive for the deposit and dissemination of scientific research documents, whether they are published or not. The documents may come from teaching and research institutions in France or abroad, or from public or private research centers.
L'archive ouverte pluridisciplinaire HAL, est destinée au dépôt et à la diffusion de documents scientifiques de niveau recherche, publiés ou non, émanant des établissements d'enseignement et de recherche français ou étrangers, des laboratoires publics ou privés. 


\title{
Elastic surface waves in crystals. Part 1: Review of the physics
}

\author{
Nathalie Favretto-Cristinia ${ }^{\mathrm{a}, *}$, Dimitri Komatitsch ${ }^{\mathrm{b}, 1}$, José M. Carcione ${ }^{\mathrm{c}}$, Fabio Cavallinic \\ ${ }^{a}$ Laboratoire de Mécanique et d'Acoustique (UPR 7051), CNRS, 31 chemin Joseph Aiguier, 13402 Marseille \\ Cedex 20, France. \\ ${ }^{b}$ Géosciences Environnement Toulouse, (UMR 5563) UR 154 CNRS Université Paul Sabatier, Observatoire \\ Midi-Pyrénées, 14 avenue Édouard Belin, 31400 Toulouse, France. \\ ${ }^{c}$ Istituto Nazionale di Oceanografia e di Geofisica Sperimentale, Borgo Grotta Gigante 42c, 34010 Sgonico,
} Trieste, Italy.

\begin{abstract}
We present a review of wave propagation at the surface of anisotropic media (crystal symmetries). The physics for media of cubic and hexagonal symmetries has been extensively studied based on analytical and semi-analytical methods. However, some controversies regarding surfaces waves and the use of different notations for the same modes require a review of the research done and a clarification of the terminology. In a companion paper we obtain the full-wave solution for the wave propagation at the surface of media with arbitrary symmetry (including cubic and hexagonal symmetries) using two spectral numerical modeling algorithms.
\end{abstract}

Keywords: Surface waves, anisotropy

\section{Introduction}

The problem of surface wave (also called surface acoustic wave - SAW) propagation in anisotropic media has been studied for many decades. It is of interest in acoustics of materials, e.g., non-destructive testing of materials, where the anisotropic elastic properties of thin coatings or media containing subsurface cracks may be evaluated from measurements of the characteristics of laser-generated SAW [1-6], in exploration geophysics [7-9], and in seismology [10-13].

A homogeneous isotropic elastic half-space can accomodate three types of bulk waves: the P, $\mathrm{SV}$ and SH waves, whose polarization vector is either parallel (for the $\mathrm{P}$ wave) or perpendicular (for the two $\mathrm{S}$ waves) to the slowness vector. The polarization of the $\mathrm{S}$ waves with respect to the free surface of the half-space is either vertical (for the SV wave) or horizontal (for the SH wave). Only the P-wave and the SV-wave may be coupled at the flat free surface of homogeneous isotropic elastic solids. A Rayleigh-type SAW resulting from the linear combination of the inhomogeneous $\mathrm{P}$ - and S-waves then travels along the free boundary of an isotropic half-space. It propagates without dispersion with a constant velocity close to the S-wave velocity of the medium [14,

\footnotetext{
${ }^{*}$ Corresponding author

Email addresses: favretto@lma.cnrs-mrs.fr (Nathalie Favretto-Cristini), dimitri.komatitsch@get.obs-mip.fr (Dimitri Komatitsch), jcarcione@inogs.it (José M. Carcione), fcavallini@inogs.it (Fabio Cavallini)

URL: http://www.univ-pau.fr/ dkomati1 (Dimitri Komatitsch)

${ }^{1}$ Also at Institut Universitaire de France, 103 boulevard Saint-Michel, 75005 Paris, France. Formerly at Université de Pau et des Pays de l'Adour, CNRS and INRIA, Laboratoire de Modélisation et d'Imagerie en Géosciences (UMR 5212) and IPRA, Avenue de l'Université, 64013 Pau Cedex, France.
} 
15]. The energy carried by this SAW, whose polarization is elliptical in the sagittal plane, is concentrated in a one-wavelength-thick waveguide just below the free surface [16]. A point source radiates, in addition to the $\mathrm{P}, \mathrm{S}$ and Rayleigh wavefronts, an $\mathrm{S}$ head wave that connects the $\mathrm{P}$ and $\mathrm{S}$ waves $[17,18]$.

In the presence of anisotropy, wave propagation presents substantial differences compared to the isotropic case. The polarization vector of the three bulk waves is not necessarily parallel nor normal to the slowness vector. Except for specific propagation directions, the "quasi" P- and the two "quasi" S-waves may be coupled at the boundary of the elastic half-space and, contrary to the isotropic case, the SAW in anisotropic media may then result from the linear combination of three or two inhomogeneous bulk waves, or even from only one inhomogeneous bulk wave. The anisotropic behavior of the medium therefore considerably modifies the existence and the structure of the SAW that propagates at the free surface of the medium. The most striking change is that an anisotropic half-space, contrary to the isotropic case, can accommodate various supersonic SAWs (i.e., SAWs with at least one homogeneous bulk wave component), as will be shown below. Moreover, the properties of the SAW are mainly constrained by the orientation of the free surface and by the direction of propagation.

Anisotropy therefore induces considerable difficulties in analytically and explicitly studying wave propagation. Attempts to derive explicit secular equations have been reported in [19-27], but most of the methods used are only applicable to specific anisotropic media with a high level of symmetry and may have spurious roots that must be carefully analyzed and suppressed. Few problems in elastodynamics have a closed-form analytical solution and some can be investigated with semi-analytical methods, but often one cannot be sure if these methods give reliable solutions. Being able to accurately simulate wave propagation numerically is therefore essential in a wide range of fields, including ultrasonics, earthquake seismology and seismic prospecting where the systems generally possess anisotropic properties, described, in their most general form, by 21 elastic coefficients and by the mass density of the material.

In the following sections we review many aspects of harmonic and transient wave propagation in anisotropic media. In a companion paper [28] we use two full-wave numerical methods to solve the problem without any approximation regarding the type of symmetry nor the orientation of the free surface.

\section{Harmonic fields}

A survey of the extensive literature shows that most theoretical and experimental studies on SAW propagation in anisotropic solids have been conducted in the frequency domain, which allows a deeper understanding of the physical phenomena involved. Unfortunately the various surface waves are often given different names in different articles for the same kind of waves. In appendix A we therefore review the different surface waves that can be present in anisotropic media, and the results obtained in previous works.

To our knowledge, SAWs in an anisotropic half-space were first studied by Sveklo [29], Stoneley [30], Gold [31], Deresiewicz and Mindlin [32], and Synge [33], followed by Gazis et al. [34], Buchwald [35] and Buchwald and Davies [36]. After deriving a complex secular equation and solving it by discarding some valid solutions, such as those associated with the generalized Rayleigh wave for instance (see more comments in Burridge [37] and Musgrave [38]), most of the authors concluded incorrectly that SAWs could travel either only in discrete directions, or in some well-defined regions, along symmetry planes of certain cubic materials. Later, Lim and Farnell [39] made extensive numerical computations and could not find the previously reported range of "forbidden" directions of SAW propagation along symmetry as well as non-symmetry planes of various crystals. This result was also confirmed by the thorough mathematical analysis of the 
complex secular equation presented in Burridge [37]. Moreover, Lim and Farnell [39, 40] studied numerically the range of existence and the behavior of the various waves that can propagate at the free surface of cubic crystals. By analyzing the roots of the secular equation in the complex plane, they deduced the velocity, attenuation, displacement, and energy flow of the large variety of "surface waves" for any direction of propagation at the (001), (110), and (111) planes of cubic media. The review of Farnell [41] is an excellent concise survey of harmonic propagation of SAWs at the free surface of cubic media.

These results obtained based on computer studies have been strengthened by theoretical foundations making use of the so-called "sextic" formalism. This approach, now known as the Stroh formalism, stems from the analogy between elastic fields created by uniformly moving line dislocations and surface wave propagation, initially recognized by Stroh [42], and subsequently extended by many researchers, including Barnett, Lothe and their collaborators [43-45]. By expressing the equation of motion and the stress-strain law as a linear system of six first-order ordinary differential equations with respect to the six-dimensional polarization-traction vector, the sextic state-vector formalism reformulates the SAW problem into a standard eigenvalue problem $[46,47]$. The six eigenvectors represent six inhomogeneous waves. The three eigenvectors that are associated with physical solutions are directly related to the waves whose linear combination results in SAW solutions (see Barnett [48] for a concise description of the Stroh formalism for steady waves). Under the framework of the Stroh formalism, the criteria for the existence of the different kinds of waves that can propagate at the free surface of an anisotropic elastic half-space have been thoroughly discussed in many articles, and general theorems have been established.

Limiting bulk waves (LBW) are waves propagating along the surface of an anisotropic halfspace with threshold velocities, namely the so-called limiting velocities. These waves exist for any crystallographic orientation, and their velocity can easily be determined geometrically from the analysis of the cross-section of the slowness surfaces of the three bulk waves at the sagittal plane. The successive limiting velocities are given by the tangential contact between the slowness branches and the normal to the free surface. The limiting velocities are therefore the lowest velocities for bulk wave propagation and the LBWs carry energy along the surface [49]. In an isotropic medium the velocity of the slowest $\mathrm{LBW}$ is just the S-wave velocity. The velocity $V_{\text {lim }}$ of the slowest LBW plays a key role in the general theory of SAW propagation in anisotropic media, as it constitutes the transition between the subsonic region, where wave velocities $V$ are smaller than $V_{\lim }$ and the eigenvalues of the SAW problem are three pairs of complex conjugates, and the supersonic region where wave velocities $V$ are higher than $V_{\lim }$. At the limit $V_{\lim }$, called the first transonic state [48], one of the conjugate pairs coalesces into one degenerate eigenvalue that in the supersonic region splits into a pair of different real eigenvalues.

The exceptional limiting bulk waves (EBW), also improperly called the exceptional bulk waves (see Appendix A), also play a central role, as will be shown below. Such waves are in fact particular LBWs whose polarization vector always lies in the plane that bounds the anisotropic medium, and which satisfy the boundary conditions at the surface of the anisotropic half-space. In that sense, they are similar to the so-called surface skimming bulk wave in isotropic half-spaces $[48,50,51]$, the SH wave being a particular example of such a surface skimming bulk wave, and then a particular case of EBW. Nevertheless, contrary to the isotropic case, EBWs can be either quasi S-waves or quasi P-waves, and they are truly "exceptional" in the sense that they occur only for isolated directions of propagation on certain orientations of the crystal surface. For very simple cases such as high symmetry configurations (e.g., the [100] or [110] directions on the (001) surface of a cubic crystal), they are just an SH type.

In anisotropic media there is a large variety of SAWs that can be either subsonic or supersonic, contrary to the isotropic case. Two types of classifications have been proposed. The first is based 
on the degeneracies in the Stroh formalism [52-55], while the second is formulated in terms of the number of inhomogeneous (and homogeneous) bulk waves involved in the construction of the SAW solution. In the more general case when the free surface is not a symmetry plane of the anisotropic material, the SAW consists of a linear combination of three inhomogeneous waves. Yet, following the behavior of the roots of the characteristic equation and the value of the coefficients of the linear combination, the SAW can be composed of three, two, or even simply one inhomogeneous wave, contrary to the isotropic case. The SAWs are thus classified into three types: three-component, two-component, and one-component SAW. For instance, for some specific directions of propagation, such as symmetry planes, one bulk wave is not coupled with the two others, and only two of the three bulk waves are therefore involved in the construction of the SAW. As an example, one can mention the subsonic two-component SAW, which cannot be polarized parallel to the free boundary plane in stable anisotropic linear elastic half-spaces [56]. It has been proven that there exists at most one such "pure" SAW solution in the subsonic domain for a given geometry of propagation. As for the isotropic case, the pure SAW results from a linear combination of only two inhomogeneous bulk waves whose amplitude decays exponentially and monotonically away from the surface. This subsonic two-component Rayleigh wave (RW) exists, provided an EBW does not exist, otherwise a subsonic two-component generalized RW (see Appendix A), whose amplitude decays exponentially but with oscillations away from the surface, may or may not arise $[45,46,57,58]$.

A SAW undamped in the direction of propagation is intrinsically a subsonic phenomenon. Nevertheless, contrary to the isotropic case, pure undamped two-component SAWs can propagate with supersonic velocity for some specific directions of propagation on the free surface of materials of various degrees of symmetry. The symmetrical supersonic surface waves (symmetrical SSW) (see Appendix A) are the first kind of two-component waves that can exist in the supersonic region. They can occur in so-called $\alpha$-configurations [59-62], in which the so-called reference plane $\Re$, spanned by the normal $\hat{\mathbf{n}}$ to the surface and the propagation direction $\hat{\mathbf{m}}$, coincides with a plane of material symmetry [63]. For instance for cubic materials the symmetrical SSW can only exist on the (001) and (110) planes [64]. For high-symmetry directions, such as the [110] direction on the (001) plane of a cubic crystal, such a wave is simply a supersonic SAW of generalized-Rayleigh type. A pure undamped (two-component) generalized Rayleigh wave may propagate with supersonic velocity for isolated cases usually corresponding to high-symmetry directions of propagation in which an EBW exists [41]. This wave, which can be considered as secluded from the subsonic branch of the SAW, is in fact a starting point for the branch of a leaky $S A W$ [65-67].

For off-symmetry directions, the elastodynamics equations of motion having both inhomogeneous and homogeneous wave solutions, the (three-component) leaky SAW radiates its energy towards the bulk of the anisotropic medium (see Appendix A), as the leaky Rayleigh waves do at specific liquid-solid interfaces [68]. The velocity of the leaky SAW and the magnitude of its attenuation along the direction of propagation strongly depend on the perturbation of the orientation from the symmetry direction [69], but for many cases the radiation of energy is small enough that the leaky SAWs are easily observable in experimental conditions [70, 71]. As the orientation of the direction of propagation approaches high-symmetry directions, the contribution of the bulk homogeneous wave in the construction of the SAW may however vanish, together with the damping of the leaky SAW in the direction of propagation, and the (three-component) leaky SAW turns into a generalized RW. The leaky SAW can originate either from a (two-component) generalized RW (as is the case for the (001) plane of copper [41]), or from an EBW [72] by a resonance phenomenon [73, 74] (as is the case for the (111) plane of copper [41]). Note that solutions of leaky-SAW type occur neither in isotropic media, nor in weakly anisotropic media (see Farnell [41], page 164). Along the leaky SAW branch there may exist other isolated points where 
the contribution of the bulk homogeneous wave in the construction of the SAW may vanish, together with the damping of the leaky SAW [75, 76], leaving a pure undamped two-component (non symmetrical) supersonic SAW. This wave can be considered as secluded from the branch of the subsonic SAW, and it is then called the secluded supersonic surface wave (secluded SSW [77-79]) (see Appendix A).

The existence and the properties of SAW in anisotropic media are mainly constrained by the orientation of the free surface and by the direction of propagation. This fact is well illustrated by the secluded (non symmetrical) SSW. Indeed, this wave cannot propagate in the $\alpha$-configuration $[63,67,76]$. Nevertheless, this kind of wave may propagate when the free boundary of the anisotropic half-space is a symmetry plane (the so-called $\beta$-configuration [63]), or when the plane perpendicular to the reference plane and to the direction of wave propagation is a symmetry plane (the so-called $\gamma$-configuration $[63,67,76]$ ). The secluded SSW generally exists for a direction for which there is also a subsonic RW [77]. This is precisely the case, for instance, for the (001) plane of cubic crystals [67]. Its occurrence has been investigated in a more general case by Maznev and Every [79] for a germanium crystal. It has been found to exist for a one-dimensional subspace, within the three-dimensional space of SAW geometries (i.e. surface orientations and propagation directions).

Contrary to the subsonic domain, a one-component $S A W$ (see Appendix A), consisting of one inhomogeneous wave that satisfies the boundary condition at the surface of the anisotropic half-space, can exist in the supersonic region in an arbitrarily anisotropic crystal under certain conditions depending on the value of the elastic coefficients of the medium $[60,80]$. General existence theorems for such waves that do not exist in isotropic materials have also been established for anisotropic media with symmetry in many studies. No one-component SAW generally exists in $\alpha-, \beta-$, or $\gamma$-configurations [60, 81, 82], except for particular triclinic materials [80] and for some orthorhombic and some transversely-isotropic (TI) materials [83]. More specifically, a one-component SAW cannot travel in stable TI media whose symmetry axis coincides with the direction of propagation $[81,84]$. It has also been shown that no one-component SAW can exist in stable cubic materials $[81,84]$.

In the subsonic region, a kind of SAW arises in the neighborhood of the directions of propagation that permit the existence of an EBW [49] and therefore, following the condition for the existence of RW established by Barnett and Lothe [44], in the neighborhood of orientations for which a subsonic RW cannot propagate [85]. The so-called quasi-bulk surface wave (QBSW) (see Appendix A) can be viewed as a continuous transition from the RW towards the EBW, through non-pure SAW with particle displacements not occurring in the sagittal plane and energy flux not being collinear with the propagation vector. Near the symmetry directions where an EBW can propagate, the SAW is transformed into a QBSW with a depth of penetration that increases for decreasing angles of deviation from the symmetry directions. The penetration of the wave into the crystal may become so large that the term "surface wave" can be misleading. In fact, the QBSW results from the (very) small contribution of two inhomogeneous waves and the predominant contribution of one wave whose characteristics differ slightly from those of the EBW. The properties of the QBSW, which are thus strongly related to the associated EBW, have been studied for crystals of hexagonal [85] and arbitrary [86] symmetries. In particular the phase velocity of the QBSW is found to be slightly smaller than the associated limiting velocity of the bulk waves.

Many authors have emphasized the close connection between the space of simple reflection and the space of degeneracy in the Stroh eigenvalue problem and in SAW problems [41, 53, 69, 76$78,83,87,88]$. 


\section{Transient propagation}

Whereas harmonic wave propagation at the free surface of anisotropic media has been widely investigated, research on transient wave propagation is rather scarce. The so-called Lamb's problem [89] is the study of the response of an elastic half-space to an impulsive line or point load at its surface. The fundamental solution for a homogeneous isotropic elastic material, both in the frequency and in the time domains, is classical $[89,90]$. Unfortunately, anisotropy introduces considerable difficulty in generalizing this solution. A convenient way of deriving the displacement Green's functions of anisotropic materials is to use integral transform techniques, and more specifically mixed Fourier-Laplace transforms. Several researchers have used the Cagniard-de Hoop (CdH) method [91-93] to reduce the Fourier-Laplace transforms to a single integral over a contour in a complex plane that must be determined. Kraut [94] first applied this method to study the 2D Lamb's problem for a line source of normal stress lying on the free surface of a TI medium (a beryl crystal) normal to the axis of symmetry. Burridge [95] extended the technique to the most general class of anisotropic solids and to a surface of arbitrary orientation loaded by an impulsive line traction. Only numerical results were presented for the surface displacement at the (111) plane of cubic copper, and the RW and leaky SAW were identified. The case of a point source, applied at the surface of TI solids with its normal coinciding with the axis of symmetry, has been treated in a similar way by Ryan [96]. In contrast with the 2D problem, the 3D Lamb's problem does not admit an explicit solution for the surface displacements. They are then found in terms of single finite integrals that must be evaluated numerically. In Payton [97], 2D and 3D problems of transient wave propagation in TI half-spaces that admit an explicit representation of the displacement field are studied. In particular, the epicenter motion of the surface due to a buried point source located on the symmetry axis, and the epicentral-axis motion caused by a normal point load suddenly applied on the surface, are explicitly evaluated based on residue calculations in the complex plane. They are also shown to be related by the BettiRayleigh theorem. More recently, Deschamps and his co-authors used the $\mathrm{CdH}$ technique to calculate the interior and the surface responses of a general cubic half-space to line and point loadings [98-101]. By analyzing the $\mathrm{CdH}$ contours and the singular points in the complex plane, they observed physical phenomena such as wavefront focusing for both the RW and the EBW as well as the diffraction caused by the cusps and the possible generation of the leaky SAW. Numerical calculations of only the normal component of the displacement (used in laboratory laser experiments) have been performed for a half-space belonging to the cubic class of symmetry. For instance, results for the (100) surface of a copper crystal and several directions of observation are shown in Bescond and Deschamps [100, 101].

As for the anisotropic case the $\mathrm{CdH}$ technique requires the analysis of complicated branch-cut integrals in the complex plane, it is considered too cumbersome to be numerically handled and therefore alternative methods have been proposed. One of them, first developed by Willis [102] and then used by Wang and Achenbach [103-106], uses Fourier and Radon transforms. It is based on a direct construction of the solution to the 3D Lamb's problem for general anisotropic solids by a superposition of time-transient plane waves. It allows one to obtain integral expressions defined in a finite domain that corresponds to the projection of the slowness surface to the surface of the solid. Unfortunately, their calculations have not emphasized the cuspidal structure in the Rayleigh wavefront, as well as the existence of the leaky SAW. In a similar way, Tewary and Fortunko [107] derived an expression for the 3D waveforms due to a delta-function pulse on the free surface of tetragonal solids, convenient for numerical computations as it requires only a 1D numerical integration. Another alternative method to the $\mathrm{CdH}$ technique is based on Fourier transforms of the equations of motion and boundary conditions, with respect to the time and the spatial coordinates parallel to the surface. The surface displacement response of 
an anisotropic half-space to sudden loading at a point on the surface is then reduced to a 1D integral for numerical evaluation [108]. The method used by Every and his collaborators can cope with Rayleigh poles and leaky SAW resonances, as shown by the good agreement between the calculated surface displacement responses to a point loading for several directions on the (001)-oriented surface of copper crystal and measured surface waveforms [109]. More precisely, multiple RW arrivals resulting from the folding of the SAW ray surface associated with sharp peaks in the SAW amplitude expressing caustics in the SAW intensity, and then the so-called "phonon focusing effect" [110-115], have been predicted and observed experimentally, as well as leaky SAW resonance and singularities in bulk wave arrival associated with the presence of EBW. In the case of a general anisotropic half-space subjected to an impulsive line load, Maznev and Every [116] derived results similar to those reported by Burridge [95] by using Fourier transforms. They illustrated the role of SAW, leaky SAW and bulk waves in the calculated surface response for the (111)-oriented surface of silicon. The studies providing the dynamic displacement response of the (001)-, (110)-, and (111)-oriented surface of copper crystal, developed by [5, 100, 101, 109], will be used as a reference in our numerical study reported in a companion paper.

\section{Conclusions}

We provided a review of harmonic and transient elastic wave propagation in anisotropic media with particular emphasis on surface-wave propagation in crystals, minerals and metals. This review clarifies the terminology used for the surface waves. In a companion paper we propose two spectral numerical modeling algorithms to obtain the full-wave solution for the wave propagation at the surface of media with arbitrary symmetry.

\section{Acknowledgements}

The authors thank Alexander Shuvalov and Arthur G. Every for fruitful discussion, and an anonymous reviewer for suggesting additional references.

This material is based in part upon research supported by European FP6 Marie Curie International Reintegration Grant MIRG-CT-2005-017461. 


\section{Appendix A. Waves propagating at the free surface of an anisotropic half-space}

SAWs propagating along the free surface of an anisotropic elastic half-space result from the linear combination of three bulk waves, which are generally damped with depth. The associated displacement field $\mathbf{u}(\mathbf{r}, t)$ can be written as:

$$
\mathbf{u}(\mathbf{r}, t)=\sum_{n=1}^{3} C_{n} \mathbf{A}_{n} \exp \left[i k\left(\mathbf{m} \cdot \mathbf{r}+p_{n} \mathbf{n} \cdot \mathbf{r}-V t\right)\right]
$$

where $\mathbf{r}$ is the space vector and $t$ the time variable. The coefficients $C_{n}$ of the linear combination for the three bulk waves are determined from the boundary conditions at the free surface and can be real- or complex-valued. They characterize the wave amplitude up to an arbitrary infinitesimal factor, while the vectors $\mathbf{A}_{n}$ denote their polarization. The wavenumber $k$, associated with the phase velocity $V$, is the projection of the wave vector $\mathbf{k}$ of the SAW on the direction of propagation $\mathbf{m}$ at the free surface of the anisotropic half-space. Vector $\mathbf{n}$ denotes the normal to the surface of the medium. Parameters $p_{n}=\mathbf{k} \cdot \mathbf{n} / \mathbf{k} \cdot \mathbf{m}$ are complex-valued in the subsonic region, while in the supersonic region at least one $p_{n}$ lies on the real axis of the complex plane and is associated with a bulk wave. Depending on the values of $C_{n}$ and $p_{n}$, the SAW consists of one, two, or three components. There is a large variety of SAWs that can propagate at the free surface of anisotropic materials, therefore let us review them here.

\section{Appendix A.1. Exceptional (limiting) bulk wave (EBW)}

Strictly speaking, the exceptional bulk waves (EBW) $[46,117]$ are bulk waves that satisfy the boundary conditions at the free surface of the anisotropic medium, and whose polarization vector always lies in the plane that bounds the medium [118]. Unlike the limiting bulk waves (LBW), these waves are in fact eigensolutions for the SAW problem. In most articles the term EBW is however improperly used for the particular EBW that propagates with the limiting velocity $V_{\text {lim }}$, instead of the term "exceptional limiting bulk waves", although the exceptional limiting bulk waves are in fact a subset of the broader class of EBW. The exceptional limiting bulk waves then combine the properties of the EBW (namely, polarization parallel to the free surface of the medium, and boundary conditions satisfied at the free surface) with those of the limiting bulk waves (namely, propagation at the limiting velocity, and energy flux parallel to the crystal surface [45]). Hereafter and in the article, the exceptional limiting bulk waves are denoted by EBW. Though propagating along the surface, unlike SAWs the EBWs do not decrease in amplitude as the distance from the surface increases. Their presence is a general feature of general anisotropic crystals, and it is not limited to media with high symmetry only [118-121]. The EBWs are quite similar to the so-called surface skimming bulk waves in isotropic solids [48, 50, 51], SH waves being a particular case of such surface skimming bulk waves. Nevertheless, unlike these waves, EBWs are not limited to definite kinds of bulk waves [48]: they can be quasi-P or quasi-S waves, provided that their polarization vector lies in the plane that bounds the medium. While quasi-S EBWs exist in all crystals, only few anisotropic media permit the propagation of quasi-P EBWs [51]. Moreover, EBWs satisfy the boundary conditions at the surface of the anisotropic half-space only for specific directions.

EBWs are also termed "improper SAWs", "bulk surface waves" [45], or even "surface skimming bulk waves" $[48,50,109,122]$. The term "lateral waves" is also used [4] and should be understood as "grazing rays". The EBW should not be mistaken for head waves.

Composite EBWs [57] that are a superposition of two or three EBWs can exist for certain types of transonic states, according to the classification established by Chadwick and Smith [46] (see Barnett [48] for a detailed discussion). Nevertheless, the EBW of the first kind described in 
Chadwick and Smith [46] and that is not composite is in fact the usual EBW defined in SAW theory.

\section{Appendix A.2. Rayleigh wave (RW)}

For an arbitrary free boundary surface and an arbitrary direction of propagation, the Rayleigh wave consists of a linear combination of three phase-matched evanescent waves whose amplitude decays exponentially and monotonically away from the surface. The RW is thus intrinsically a subsonic phenomenon. It then propagates undamped at the free surface of an elastic half-space with a subsonic velocity $V_{R}$ that is slightly smaller than the limiting velocity $V_{\lim }$. The motion of the RW describes an ellipse that is generally tilted with respect to the sagittal plane. However, for symmetry directions, only two of the three waves are involved in the RW construction and the corresponding motion describing an ellipse occurs in the sagittal plane, as in the isotropic case. The energy carried by the RW is generally concentrated in a roughly one-wavelength-thick waveguide below the free surface for high symmetry directions, but it can penetrate deeper in the bulk of the anisotropic medium for out-of-symmetry directions. The RW never coexists with an EBW propagating in the same direction, as in the isotropic case.

Because its properties are similar to those of a RW propagating at the free surface of an isotropic elastic medium, the RW is also called an "ordinary RW" [123], a "pure SAW" or "pure RW", or a "proper SAW" [44].

\section{Appendix A.3. Generalized Rayleigh wave (generalized $R W$ )}

The generalized Rayleigh wave is a kind of Rayleigh wave that can propagate with either a subsonic or supersonic velocity. It does not exist in isotropic media. Unlike the RW, it consists of two components whose amplitude is non-monotonically damped towards the bulk of the anisotropic medium, but with an oscillatory trend $[123,124]$. The period of the oscillations of the amplitude as well as the penetration distance in the bulk of the medium and the velocity of the generalized RW depend on the so-called anisotropy parameter of the material. For instance, for the (001) plane of a cubic medium this parameter is characterized either by [125] $A=$ $2 c_{44} /\left(c_{11}-c_{12}\right)$, or by [123] $\eta=1 / A$, where $c_{11}, c_{44}$, and $c_{12}$ are the three independent elastic coefficients of the anisotropic medium. It has been shown that the period of the oscillations decreases with increasing values of the anisotropy parameter [125] $A$, or decreasing values of parameter [123] $\eta$. Moreover, the velocity of the generalized RW decreases with decreasing values of parameter $\eta$. Approximate expressions for the phase velocity and the oscillation damping have been established for cubic media with $\eta<\frac{1}{4}$ in Royer and Dieulesaint [125]. Usually, the generalized RW propagates undamped in the direction of propagation with a subsonic velocity and its polarization is elliptical in the sagittal plane. Nevertheless, the generalized RW can also be a two-component supersonic wave for isolated cases corresponding usually to high-symmetry directions of propagation [41]. In this case, it generally belongs to the branch of a leaky SAW $[65,76]$. Contrary to the RW, the generalized RW may coexist with an EBW propagating in the same direction.

The RW and the generalized RW cannot coexist. For instance, for cubic materials and depending on the anisotropy parameter $\eta$, the RW that propagates along symmetry directions on high-symmetry boundary surfaces can be either ordinary or generalized. Kosevich et al. $[123,124,126]$ have analyzed the conditions under which the transition from an ordinary RW to a generalized RW takes place in cubic crystals for various values of the anisotropy parameter $\eta$. Their work strengthens the numerical study of Gazis et al. [34]. The transition occurs at a particular value $\left(\eta=\eta_{0} \simeq 1\right)$ for which there is degeneracy of the roots of the characteristic equation for the bulk vibrations. The region $\eta<\eta_{0}$ corresponds to the existence of the generalized $\mathrm{RW}$, while the region $\eta>\eta_{0}$ corresponds to the existence of the ordinary RW. It has been shown 
[124] that the non-convexity of the cross-section of the slowness surface of the bulk shear wave, polarized in the sagittal plane, is a sufficient but not a necessary condition for the existence of a generalized RW. In highly anisotropic crystals (i.e., for instance with $\eta \ll 1$ ), one of the two components involved in the construction of the generalized RW can become dominant, have an almost linear polarization normal to the free surface, and can penetrate into the crystals to a depth significantly greater than the wavelength. The generalized RW can thus possess the properties of both the bulk wave and the pure RW, whose penetration depth is about the wavelength. Such a wave is called the deeply penetrating RW (DPRW) and has been thoroughly investigated by Kosevich et al. [126] in the case of wave propagation along the [100] direction on the (001) plane of a cubic crystal. However, the DPRW can propagate in crystals characterized by a strong anisotropy of the velocity of bulk shear waves polarized in other sagittal planes as well [124].

The generalized RW is also sometimes called "Rayleigh wave" by Lothe and his collaborators, which may be confusing.

\section{Appendix A.4. Quasi-bulk surface wave (QBSW)}

The quasi-bulk (surface) wave arises in the neighborhood of the direction of propagation at which an EBW exists. The criterion for the existence (respectively, absence) of the QBSW in the neighborhood of symmetry orientations is then the absence (respectively, existence) of subsonic RWs propagating in the symmetry direction [85]. At small deviation from this symmetry direction, the bulk wave associated with the EBW is dominant in the construction of a threecomponent SAW and the three partial wave combination as a whole is referred to as a QBSW. In fact, the QBSW can be considered as a gradual change of the SAW into the EBW. Its polarization vector tilts towards the free surface of the anisotropic half-space. Furthermore, the smaller the angle of deviation from the symmetry orientation, the less damped the wave is into the medium, i.e., the wave penetrates deeper.

This is the reason why the QBSW is also called "quasi-bulk Rayleigh wave" [86], or "deeply penetrating Rayleigh wave" [5], which may lead to confusions with the terminology used by Kosevich and his collaborators to describe the behavior of RW in crystals with strong anisotropy [126].

\section{Appendix A.5. Leaky surface acoustic wave (leaky SAW)}

The leaky wave is a solution to the boundary-value problem in the form of a linear combination of two inhomogeneous waves and one bulk wave that radiates energy into the anisotropic medium. The leaky SAW is inherently a supersonic phenomenon, and its velocity lies between the first and second limiting velocities. This wave propagates damped in the direction of propagation. Nevertheless, as for many cases the radiation of energy is small enough, the leaky SAW can easily be observable in experimental conditions [70,71]. Its properties are quite similar to those of a leaky SAW propagating at the interface between an ideal fluid and an isotropic elastic medium $[68,127]$. Note that this kind of wave does not exist in an isotropic half-space. Approximate expressions for its velocity and the magnitude of its attenuation along the direction of propagation are given without any assumption on the symmetry of the crystal in [69]. The leaky SAW can be caused by a generalized RW or by an EBW [69, 72, 75].

The leaky SAW is also called "pseudo SAW" [85], or "supersonic wave".

\section{Appendix A.6. Secluded supersonic surface wave (secluded SSW)}

The secluded supersonic surface wave exists at the so-called pure points where the damping of the leaky wave in the direction of propagation vanishes together with the coefficient of the linear combination associated with the bulk wave. This results in a two-component (non-symmetrical) 
supersonic surface wave that generally exists for a specific direction along which a subsonic Rayleigh wave can also propagate [77-79]. It can be seen as due to confluence between the space of simple reflection and the leaky SAW branch [67, 76, 83, 128].

The secluded SSW is also called "non symmetrical supersonic wave" [79].

\section{Appendix A.7. One-component surface acoustic wave (one-component SAW)}

Mathematically, it is represented by degeneracy in the Stroh eigenvalue problem [60]. The onecomponent surface wave consists of only one inhomogeneous wave and is necessarily supersonic. There are three versions of the one-component SAW [83]. Two are polarized in the plane of the boundary of the anisotropic half-space $[60,80]$. The third is a supersonic generalized onecomponent surface wave similar to the generalized Rayleigh wave studied in Kosevich et al. [123]. No one-component SAW exists for either isotropic elastic materials, or crystals of cubic symmetry $[81,84]$. This kind of wave can occur under certain conditions for particular triclinic media [80] as well as for some particular TI media [81, 83, 84]. The one-component SAW can be seen as due to confluence between the space of degeneracy and a two-component secluded SSW [83].

\section{Appendix A.8. Symmetrical surface waves (symmetrical SAW)}

Symmetrical surface waves are necessarily two-component SAWs that can occur in both the subsonic and the supersonic regions when the sagittal plane in which they are polarized is a plane of symmetry of the material $[59,60,66]$. They form a continuous branch extending from the subsonic region into the supersonic region and occurring in materials of monoclinic, orthorhombic and cubic symmetry $[61,62]$. For high-symmetry directions on symmetry planes, such waves are typically of RW or generalized-RW type.

\section{References}

[1] B. Castagnède, Y. Berthelot, Photoacoustic interactions by modulation and laser impact: applications in mechanics and physics of anisotropic solids, Journal d'Acoustique 5 (1992) 417-453.

[2] J. F. Chai, T. T. Wu, Determination of anisotropic elastic constants using laser-generated surface waves, J. Acoust. Soc. Am. 95 (1994) 3232-3241.

[3] D. C. Hurley, V. K. Tewary, A. J. Richards, Surface acoustic wave methods to determine the anisotropic elastic properties of thin films, Measurement Science Technology 12 (2001) $1486-1494$.

[4] A. G. Every, Measurement of the near-surface elastic properties of solids and thin supported films, Measurement Science Technology 13 (2002) R21-R39.

[5] A. G. Every, M. Deschamps, Principal surface wave velocities in the point focus acoustic materials signature $\mathrm{V}(\mathrm{z})$ of an anisotropic solid, Ultrasonics 41 (2003) 581-591.

[6] K. van Wijk, D. Komatitsch, J. A. Scales, J. Tromp, Analysis of strong scattering at the micro-scale, J. Acoust. Soc. Am. 115 (3) (2004) 1006-1011. doi:10.1121/1.1647480.

[7] J. M. Carcione, Domain decomposition for wave propagation problems, J. Sci. Comp. 6 (4) (1991) 453-472.

[8] S. Hestholm, B. Ruud, 2D finite-difference elastic wave modelling including surface topography, Geophys. Prospect. 42 (1994) 371-390. 
[9] E. Tessmer, D. Kosloff, 3-D elastic modeling with surface topography by a Chebyshev spectral method, Geophysics 59 (3) (1994) 464-473.

[10] M. D. Trifunac, Surface motion of a semi-cylindrical alluvial valley for incident plane $S H$ waves, Bull. Seismol. Soc. Am. 61 (1971) 1755-1770.

[11] M. Bouchon, Effect of topography on surface motion, Bull. Seismol. Soc. Am. 63 (1973) $615-632$.

[12] S. Crampin, D. B. Taylor, The propagation of surface waves in anisotropic media, Geophys. J. Int. 25 (1-3) (1977) 71-87.

[13] S. J. Lee, H. W. Chen, Q. Liu, D. Komatitsch, B. S. Huang, J. Tromp, Three-dimensional simulations of seismic wave propagation in the Taipei basin with realistic topography based upon the spectral-element method, Bull. Seismol. Soc. Am. 98 (1) (2008) 253-264. doi:10.1785/0120070033.

[14] L. Knopoff, On Rayleigh wave velocities, Bulletin of the Seismological Society of America 42 (1952) 307-308.

[15] L. Landau, E. Lifchitz, Théorie de l'élasticité (Theory of Elasticity), 2nd Edition, Mir, Moscow, Russia, 1953.

[16] I. A. Viktorov, Rayleigh and Lamb waves: physical theory and applications, Plenum Press, New-York, USA, 1967.

[17] W. L. Pilant, Elastic waves in the Earth, Vol. 11 of "Developments in Solid Earth Geophysics" Series, Elsevier Scientific Publishing Company, Amsterdam, The Netherlands, 1979.

[18] J. M. Carcione, Modeling anelastic singular surface waves in the Earth, Geophysics 57 (6) (1992) 781-792.

[19] P. K. Currie, The secular equation for Rayleigh waves on elastic crystals, Quarterly Journal of Mechanics and Applied Mathematics 32 (1979) 163-173.

[20] D. B. Taylor, P. K. Currie, The secular equation for Rayleigh waves on elastic crystals. II Corrections and additions, Quarterly Journal of Mechanics and Applied Mathematics 34 (1981) 231-234.

[21] M. Destrade, The explicit secular equation for surface acoustic waves in monoclinic elastic crystals, J. Acoust. Soc. Am. 109 (4) (2001) 1398-1402.

[22] M. Destrade, Surface waves in orthotropic incompressible materials, J. Acoust. Soc. Am. 110 (2) (2001) 837-840.

[23] M. Destrade, Rayleigh waves in symmetry planes of crystals: explicit secular equations and some explicit wave speeds, Mechanics of Materials 35 (2003) 931-939.

[24] T. Ting, Explicit secular equations for surface waves in monoclinic materials with the symmetry plane $x 1=0, x 2=0$ or $x 3=0$, Proc. R. Soc. London Ser. A 458 (2002) 1017-1031. 
[25] T. C. T. Ting, Explicit secular equations for surface waves in an anisotropic elastic halfspace from Rayleigh to today, in: R. Goldstein, G. Maugin (Eds.), Surface waves in anisotropic and laminated bodies and defects detection, Kluwer Academic Publishers, Dordrecht, The Netherlands, 2004, pp. 95-116.

[26] T. C. T. Ting, The polarization vector and secular equation for surface waves in anisotropic elastic half-space, International Journal of Solids and Structures 41 (2004) 2065-2083.

[27] A. Mielke, Y. B. Fu, Uniqueness of the surface-wave speed: a proof that is independent of the Stroh formalism, Mathematics and Mechanics of Solids 9 (2004) 5-15.

[28] D. Komatitsch, J. M. Carcione, F. Cavallini, N. Favretto-Cristini, Elastic surface waves in crystals - Part 2: Cross-check of two full-wave numerical modeling methods, Ultrasonics (2011) Submitted.

[29] V. A. Sveklo, Plane waves and Rayleigh waves in anisotropic media, Doklady Akademii Nauk SSSR 59 (1948) 871-874.

[30] R. Stoneley, The propagation of surface elastic waves in a cubic crystal, Proceedings of the Royal Society of London, United Kingdom A232 (1955) 447-458.

[31] L. Gold, Rayleigh wave propagation on anisotropic (cubic) media, Physical Review 104 (6) (1956) 1532-1536.

[32] H. Deresiewicz, R. D. Mindlin, Waves on the surface of a crystal, Journal of Applied Physics 28 (6) (1957) 669-671.

[33] J. L. Synge, Elastic waves in anisotropic media, Journal of Mathematical Physics 35 (1957) 323-335.

[34] D. C. Gazis, R. Herman, R. F. Wallis, Surface elastic waves in cubic crystals, Physical Review 119 (2) (1960) 533-544.

[35] V. T. Buchwald, Rayleigh waves in anisotropic media, Quarterly Journal of Mechanics and Applied Mathematics 14 (4) (1961) 461-468.

[36] V. T. Buchwald, A. Davis, Surface waves in elastic media with cubic symmetry, Quarterly Journal of Mechanics and Applied Mathematics 16 (1963) 283-293.

[37] R. Burridge, The directions in which Rayleigh waves may be propagated on crystals, Quarterly Journal of Mechanics and Applied Mathematics 23 (2) (1970) 217-224.

[38] M. Musgrave, Crystal Acoustics - Introduction to the study of elastic waves and vibrations in crystals, Holden-Day, San Francisco, USA, 1970.

[39] T. C. Lim, G. W. Farnell, Search for forbidden directions of elastic surface wave propagation in anisotropic crystals, Journal of Applied Physics 39 (9) (1968) 4319-4325.

[40] T. C. Lim, G. W. Farnell, Character of pseudo-surface waves on anisotropic crystals, J. Acoust. Soc. Am. 45 (4) (1969) 845-851.

[41] G. W. Farnell, Properties of elastic surface waves, in: W. P. Mason, R. N. Thurston (Eds.), Physical Acoustics, Vol. 6, Academic Press, New-York, USA, 1970, Ch. 3, pp. 109-166.

[42] A. N. Stroh, Steady-state problems in anisotropic elasticity, Journal of Mathematical Physics 41 (1962) 77-103. 
[43] D. M. Barnett, J. Lothe, K. Nishioka, R. J. Asaro, Elastic surface waves in anisotropic crystals: a simplified method for calculating Rayleigh velocities using dislocation theory, Journal of Physics F: Metal Physics 3 (1973) 1083-1096.

[44] D. M. Barnett, J. Lothe, Consideration of the existence of surface wave (Rayleigh wave) solutions in anisotropic elastic crystals, Journal of Physics F: Metal Physics 4 (1974) 671686.

[45] J. Lothe, D. M. Barnett, On the existence of surface-wave solutions for anisotropic elastic half-spaces with free surface, Journal of Applied Physics 47 (1976) 428-433.

[46] P. Chadwick, G. D. Smith, Foundations of the theory of surface waves in anisotropic elastic materials, in: C. S. Yih (Ed.), Advances in Applied Mechanics, Vol. 17, Academic Press, New-York, USA, 1977, pp. 303-376.

[47] T. C. T. Ting, Anisotropic elasticity: theory and applications, Oxford University Press, United Kingdom, 1996.

[48] D. M. Barnett, Bulk, surface, and interfacial waves in anisotropic linear elastic solids, International Journal of Solids and Structures 37 (2000) 45-54.

[49] V. I. Alshits, J. Lothe, Surface waves in hexagonal crystals, Soviet Physics Crystallography 23 (1978) 509-515.

[50] S. V. Biryukov, Y. V. Gulyaev, V. V. Krylov, V. P. Plessky, Surface acoustic waves in inhomogeneous media, Vol. 20 of Springer Series on Wave Phenomena, Springer-Verlag, Berlin, Germany, 1995.

[51] N. F. Naumenko, Application of exceptional wave theory to materials used in surface acoustic wave devices, Journal of Applied Physics 79 (12) (1996) 8936-8943.

[52] T. C. T. Ting, D. M. Barnett, Classifications of surface waves in anisotropic elastic materials, Wave Motion 26 (1997) 207-218.

[53] L. Wang, Space of degeneracy in the Stroh eigensystem and surface waves in transversely isotropic elastic media, Wave Motion 40 (2004) 173-190.

[54] L. Wang, Extraordinary degeneracy and space of degeneracy in transversely isotropic elastic media, Wave Motion 45 (2008) 264-277.

[55] J. Lothe, V. Alshits, Surface waves, limiting waves and exceptional waves: David barnett's role in the development of the theory, Mathematics and Mechanics of Solids 14 (2009) $16-37$.

[56] D. M. Barnett, On the non-existence of subsonic boundary-polarized two-component free surface waves, Physica Scripta T44 (1992) 98-103.

[57] D. M. Barnett, J. Lothe, Free surface (Rayleigh) waves in anisotropic elastic halfspaces: the surface impedance method, Proceedings of the Royal Society of London, United Kingdom A402 (1985) 135-152.

[58] T. Ting, Explicit conditions for the existence of exceptional body waves and subsonic waves in anisotropic elastic solids, Wave Motion 46 (2009) 323-335. 
[59] P. Chadwick, The behaviour of elastic surface waves polarized in a plane of material symmetry. I. General analysis, Proceedings of the Royal Society of London, United Kingdom A430 (1990) 213-240.

[60] D. M. Barnett, P. Chadwick, J. Lothe, The behaviour of elastic surface waves polarized in a plane of material symmetry. I. Addendum, Proceedings of the Royal Society of London, United Kingdom A433 (1991) 699-710.

[61] P. Chadwick, N. J. Wilson, The behaviour of elastic surface waves polarized in a plane of material symmetry. II. Monoclinic media, Proceedings of the Royal Society of London, United Kingdom A438 (1992) 207-223.

[62] P. Chadwick, N. J. Wilson, The behaviour of elastic surface waves polarized in a plane of material symmetry. III. Orthorhombic and cubic media, Proceedings of the Royal Society of London, United Kingdom A438 (1992) 225-247.

[63] P. Chadwick, Wave propagation in transversely isotropic elastic media. II. Surface waves, Proceedings of the Royal Society of London, United Kingdom A422 (1989) 67-101.

[64] D. M. Barnett, S. D. Gavazza, J. Lothe, Slip waves along the interface between two anisotropic elastic half-spaces in sliding contact, Proceedings of the Royal Society of London, United Kingdom A415 (1988) 389-419.

[65] K. A. Ingebrigtsen, A. Tonning, Elastic surface waves in crystals, Physical Review 184 (3) (1969) 942-951.

[66] L. Wang, Existence of symmetric surface waves and their relation with leaky surface waves in cubic materials, Physica Scripta T44 (1992) 128-132.

[67] L. Wang, J. Lothe, Simple reflection in anisotropic elastic media and its relation to exceptional waves and supersonic surface waves: (II) examples, Wave Motion 16 (1992) 101-112.

[68] J. M. Carcione, H. B. Helle, On the physics and simulation of wave propagation at the ocean bottom, Geophysics 69 (2004) 825-839.

[69] A. N. Darinskii, On the theory of leaky waves in crystals, Wave Motion 25 (1997) 35-49.

[70] H. Engan, K. A. Ingebrigtsen, A. Tonning, Elastic surface waves in alpha-quartz: observation of leaky surface waves, Applied Physics Letters 10 (1967) 311-313.

[71] F. R. Rollins Jr, T. C. Lim, G. W. Farnell, Ultrasonic reflectivity and surface wave phenomena on surfaces of copper single crystals, Applied Physics Letters 12 (7) (1968) 236-238.

[72] A. N. Darinskii, V. I. Alshits, J. Lothe, Simple reflection and leaky waves in the vicinity of a line of exceptional bulk waves, Wave Motion 30 (1999) 253-274.

[73] A. N. Darinskii, Leaky waves and the elastic wave resonance reflection on a crystal-thin solid layer interface. II Leaky waves given rise to by exceptional bulk waves, J. Acoust. Soc. Am. 103 (1998) 1845-1854.

[74] A. N. Darinskii, M. Weihnacht, Acoustic waves in bounded anisotropic media: theorems, estimations, and computations, IEEE Transactions on Ultrasonics, Ferroelectrics, and Frequency Control 52 (5) (2005) 792-801. 
[75] G. W. Farnell, Review of pseudo-surface waves, in: Proceedings of the "Surface waves in solids and layered structures (ISSWAS86)" International Symposium, Vol. 3, Novosibirsk, Russia, 1986, pp. 7-20.

[76] L. Wang, J. Lothe, Simple reflection in anisotropic elastic media and its relation to exceptional waves and supersonic surface waves (I) General theoretical considerations, Wave Motion 16 (1992) 89-99.

[77] S. A. Gundersen, L. Wang, J. Lothe, Secluded supersonic elastic surface waves, Wave Motion 14 (1991) 129-143.

[78] J. Lothe, L. Wang, Self-orthogonal sextic formalism for anisotropic elastic media: spaces of simple reflection and two-component surface waves, Wave Motion 21 (1995) 163-181.

[79] A. A. Maznev, A. G. Every, Secluded supersonic surface waves in germanium, Physics Letters A197 (1995) 423-427.

[80] D. M. Barnett, P. Chadwick, The existence of one-component surface waves and exceptional transonic states of types 2, 4 and E1 in anisotropic elastic media, in: J. J. Wu, T. C. T. Ting, D. M. Barnett (Eds.), Modern Theory of Anisotropic Elasticity and Applications, SIAM, Philadelphia, USA, 1991, pp. 199-214.

[81] P. Chadwick, Some remarks on the existence of one-component surface waves in elastic materials with symmetry, Physica Scripta T44 (1992) 94-97.

[82] T. C. T. Ting, The motion of one-component surface waves, Journal of the Mechanics and Physics of Solids 40 (7) (1992) 1637-1650.

[83] L. Wang, S. A. Gundersen, Existence of one-component surface waves in anisotropic elastic media, Physica Scripta 47 (1993) 394-404.

[84] A. N. Norris, One-component surface waves in materials with symmetry, Journal of the Mechanics and Physics of Solids 40 (7) (1992) 1569-1582.

[85] J. Lothe, V. I. Alshits, Existence criterion for quasi-bulk surface waves, Soviet Physics Crystallography 22 (1977) 519-525.

[86] A. N. Darinskii, Quasi-bulk Rayleigh waves in semi-infinite media of arbitrary anisotropy, Wave Motion 27 (1998) 79-93.

[87] V. I. Alshits, J. Lothe, Comments on the relation between surface wave theory and the theory of reflection, Wave Motion 3 (1981) 297-310.

[88] V. I. Alshits, J. Lothe, Some basic properties of bulk elastic waves in anisotropic media, Wave Motion 40 (2004) 297-313.

[89] H. Lamb, On the propagation of tremors over the surface of an elastic solid, Phil. Trans. R. Soc. London. Ser. A 203 (1904) 1-42.

[90] J. D. Achenbach, Wave Propagation in Elastic Solids, North-Holland, Amsterdam, The Netherlands, 1973.

[91] L. Cagniard, Réflexion et Réfraction des Ondes Sismiques Progressives, Gauthiers-Villars, Paris, 1939. 
[92] A. T. de Hoop, A modification of Cagniard's method for solving seismic pulse problems, Applied Science Research B8 (1960) 349-356.

[93] J. H. M. T. van der Hijden, Propagation of transient elastic waves in stratified anisotropic media, North-Holland, Amsterdam, The Netherlands, 1987.

[94] E. A. Kraut, Advances in the theory of anisotropic elastic wave propagation, Reviews of Geophysics 1 (3) (1963) 401-448.

[95] R. Burridge, Lamb's problem for an anisotropic half-space, Quarterly Journal of Mechanics and Applied Mathematics 24 (1) (1971) 81-98.

[96] R. L. Ryan, Pulse propagation in a transversely isotropic half-space, Journal of Sound and Vibration 14 (4) (1971) 511-524.

[97] R. G. Payton, Elastic wave propagation in transversely isotropic media, Martinus Nijhoff, The Hague, The Netherlands, 1983.

[98] A. Mourad, M. Deschamps, Lamb's problem for an anisotropic half-space studied by the Cagniard-de Hoop method, J. Acoust. Soc. Am. 97 (5) (1995) 3194-3197.

[99] A. Mourad, M. Deschamps, B. Castagnède, Acoustic waves generated by a transient line source in an anisotropic half-space, Acustica - Acta Acustica 82 (1996) 839-851.

[100] C. Bescond, M. Deschamps, Dynamical surface response of a semi-infinite anisotropic elastic medium to an impulsive force, J. Acoust. Soc. Am. 103 (1) (1998) 114-124.

[101] C. Bescond, M. Deschamps, Erratum to 'Dynamical surface response of a semi-infinite anisotropic elastic medium to an impulsive force', J. Acoust. Soc. Am. 104 (1) (1998) 599.

[102] J. R. Willis, Self-similar problems in elastodynamics, Philosophical Transactions of the Royal Society of London, United Kingdom Ser. A 274 (1973) 435-491.

[103] C. Y. Wang, J. D. Achenbach, A new method to obtain 3D Green's functions for anisotropic solids, Wave Motion 18 (1993) 273-289.

[104] C. Y. Wang, J. D. Achenbach, Elastodynamic fundamental solutions for anisotropic solids, Geophys. J. Int. 118 (1994) 384-392.

[105] C. Y. Wang, J. D. Achenbach, Three-dimensional time-harmonic elastodynamic Green's functions for anisotropic solids, Proceedings of the Royal Society of London, United Kingdom A-449 (1995) 441-458.

[106] C. Y. Wang, J. D. Achenbach, Lamb's problem for solids of general anisotropy, Wave Motion 24 (1996) 227-242.

[107] V. K. Tewary, C. M. Fortunko, Surface waves in three-dimensional half-space tetragonal solids, J. Acoust. Soc. Am. 100 (1) (1996) 86-88.

[108] A. G. Every, K. Y. Kim, A. A. Maznev, The elastodynamic response of a semi-infinite anisotropic solid to sudden surface loading, J. Acoust. Soc. Am. 102 (3) (1997) 1346-1354.

[109] A. G. Every, K. Y. Kim, A. A. Maznev, Surface dynamic response functions of anisotropic solids, Ultrasonics 36 (1998) 349-353. 
[110] R. E. Camley, A. A. Maradudin, Phonon focusing at surfaces, Physical Review B 27 (4) (1983) 1959-1964.

[111] A. A. Kolomenskii, A. A. Maznev, Phonon-focusing effect with laser-generated ultrasonic surface waves, Physical Review B 48 (19) (1993) 14502-14512.

[112] A. A. Maznev, A. G. Every, Ray surface and focusing of surface acoustic waves on the basal plane of cubic crystals, Acta Acustica 1 (1994) 137-143.

[113] A. L. Shuvalov, A. G. Every, Transverse curvature of the acoustic slowness surface in crystal symmetry planes and associated phonon focusing cusps, J. Acoust. Soc. Am. 108 (5) (2000) $2107-2113$.

[114] A. Maznev, A. M. Lomonosov, P. Hess, A. A. Kolomenskii, Anisotropic effects in surface acoustic wave propagation from a point source in a crystal, The European Physical Journal B 35 (2003) 429-439.

[115] L. Wang, Caustic and anticaustic points in the phonon focusing patterns of cubic crystals, Journal of the Acoustical Society of America 123 (6) (2008) 4140-4146.

[116] A. A. Maznev, A. G. Every, Time-domain dynamic surface response of an anisotropic elastic solid to an impulsive line force, International Journal of Engineering Science 35 (4) (1997) 321-327.

[117] P. Chadwick, G. D. Smith, Surface waves in cubic elastic materials, in: H. G. Hopkins, M. J. Sewell (Eds.), Mechanics of solids, Pergamon Press, Oxford, United Kingdom, 1982, pp. $47-100$.

[118] V. I. Alshits, J. Lothe, Elastic waves in triclinic crystals: (III) The problem of existence and some general properties of exceptional surface waves, Soviet Physics Crystallography 24 (1979) 644-648.

[119] V. I. Alshits, J. Lothe, Elastic waves in triclinic crystals: (I) General theory and the degeneracy problem, Soviet Physics Crystallography 24 (1979) 387-392.

[120] V. I. Alshits, J. Lothe, Elastic waves in triclinic crystals: (II) Topology of polarization fields and some general theorems, Soviet Physics Crystallography 24 (1979) 393-398.

[121] V. I. Alshits, V. N. Lyubimov, N. F. Naumenko, N. V. Perelomova, A. L. Shuvalov, Exceptional elastic body waves in crystals of various symmetries, Soviet Physics Crystallography 30 (1985) 123-126.

[122] J. Lothe, L. Wang, Properties of Type 6 transonic states with respect to grazing incidence reflection of bulk waves at planar free or clamped surfaces of half-infinite elastically anisotropic media, Wave Motion 20 (1994) 41-56.

[123] A. M. Kosevich, Y. A. Kosevich, E. S. Syrkin, Generalized Rayleigh waves and the geometry of isofrequency surfaces of sound oscillation waves in crystals, Soviet Physics - Journal of Experimental and Theoretical Physics 61 (1985) 639-644.

[124] Y. A. Kosevich, E. S. Syrkin, A. M. Kosevich, Vibrations localized near surfaces and interfaces in non-traditional crystals, Progress in Surface Science 55 (1) (1997) 59-111.

[125] D. Royer, E. Dieulesaint, Rayleigh wave velocity and displacement in orthorhombic, tetragonal, hexagonal, and cubic crystals, J. Acoust. Soc. Am. 76 (5) (1984) 1438-1444. 
[126] Y. A. Kosevich, E. S. Syrkin, Existence criterion and properties of deeply penetrating Rayleigh waves in crystals, Soviet Physics - Journal of Experimental and Theoretical Physics 62 (6) (1985) 1282-1286.

[127] L. M. Brekhovskikh, O. A. Godin, Acoustics of layered media I, Vol. 5 of Springer Series on Wave phenomena, Springer-Verlag, Berlin, Germany, 1990.

[128] G. Stegeman, Normal-mode surface waves in pseudo-branch on (001) plane of gallium arsenide, Journal of Applied Physics 47 (1976) 1712-1713. 